\title{
Multidimensionality of tone in Pahari
}

\author{
Abdul Qadir Khan, Ph.D \\ University of Azad Jammu and Kashmir, Muzaffarabad, AJK PAKISTAN \\ $\mathrm{Yi} \mathrm{Xu}$ \\ University College London
}

\begin{abstract}
The current study investigated the contribution of different acoustic dimensions to tonal contrasts in Pahari, an understudied language in the Pakistan-administrated part of Kashmir. While previous research on the tonal languages of the region focused only on overall pitch patterns, the present study analyzed fundamental frequency $\left(\mathrm{F}_{0}\right)$, duration, intensity and phonation type. Monosyllabic lexical words that form minimal triplets with identical CV structures were recorded from eight native speakers. Detailed acoustic analyses showed that Pahari is tonal at the lexical level with three distinct tones, High, Mid and Low, characterized by high-falling, mid-level and low-rising $\mathrm{F}_{0}$ contours respectively. The study established that five acoustic cues jointly contribute to the lexical tonal contrast in Pahari. The height of $\mathrm{F}_{0}$ is the most salient and final velocity helps to distinguish the Low tone from the other two tones. Intensity and phonation help to distinguish the Low tone from the Mid tone and duration helps to distinguish the High tone from the Mid and Low tones. It is concluded that Pahari has a threeway tonal contrast realized with multiple phonetic cues. The multiplicity of cues for the tonal contrast in the language raises interesting questions about the notion of distinctive features and the principle of economy of representation.
\end{abstract}

\section{INTRODUCTION}

Traditionally, tone is defined as pitch patterns that can distinguish lexical items in a similar way to consonants and vowels (Pike, 1948). Given this definition, most research on tone has focused on pitch cues, including mean $\mathrm{F}_{0}$, direction of $\mathrm{F}_{0}$ change and slope of $\mathrm{F}_{0}$ change (Yip, 2002; Author 2, 1997). However, studies have increasingly been examining the role of other acoustic dimensions in tonal contrasts, including duration, intensity and voice quality (Brunelle, et al 2010; Kuo, Rosen and Faulkner, 2008). In particular, there is growing interest in the relationship between tone and phonation type (Gordon and Ladefoged, 2001). For example, in Mpi (Silverman, 1997) and Jalapa Mazatec (Garallek and Keating, 2011) phonation is contrastive independent of tone. In some other languages phonation is found to co-vary with certain tonal categories. For example, Mandarin Tone 3 is produced with creaky voice (BelotelGrenie and Grenie, 1994) or other phonation variants (Zheng, 2006); Vietnamese has six tones, some of which are accompanied by creaky or breathy voice (Brunelle, et al 2010). One question which is still under-investigated is whether it is possible to identify the sources of the relationship between tone and phonation. Some existing suggestions include the tendency for creaky voice to accompany a very low pitch due to the physiology of pitch production (Ladefoged, 1971). There are also proposals of voice quality implications from specific 
processes of tonogenesis, such as the suggestion that the loss of voiced aspiration has led to the development of the Low tone in Punjabi (Hombert, Ohala and Ewan, 1979). Therefore, two different phonation types are potentially associated with a low-pitched tone. The present study is an acoustic investigation of tonal contrasts in Pahari, an understudied Indo-Aryan language spoken in Pakistan, focusing in particular on the relationship between tone and voice quality.

Baart (2003) conducted a survey of 18 languages spoken in Northern Pakistan (Azad Jammu and Kashmir, Northern areas, Punjab and NWFP) and pointed out that the majority of these languages are tonal. He reported that there are approximately ninety million speakers of tonal languages in the region. He categorized these languages into three classes: 1) languages with two tones (Falling and Rising), such as Shina and Burushaski; 2) languages with three tones (High, Mid and Low), such as Punjabi and Gujrati; and 3) languages that show more than three tones, such as Kalam Kohistani. Author 1 (2017) conducted a preliminary study of Pahari tone, focusing on pitch and duration. His results showed that Pahari has a three-way tonal contrast, namely, High-falling, Mid-level and Low-rising. His results further show that the duration of the High tone is shorter than the other two tones. This suggests that $F_{0}$ is not the only acoustic dimension that helps to identify the tones in Pahari. There have been studies that show the role of other dimensions in tonal contrasts, including duration, intensity and voice quality (Brunelle, et al 2010; Kuo, Rosen and Faulkner, 2008). There is thus a possibility that these dimensions are also involved in tonal contrast in Pahari.

In particular, tonal contrast in Pahari could be due to phonation type, as in the case of Gujarati (M. Ohala, 1991), a closely related language. M. Ohala (1991) points out that the loss of aspiration in some languages has resulted in two different outcomes. In Punjabi, the voiced aspirate has developed into a 'low rising tone' (also see Gill \& Gleason,1972). But in Gujarati, a non-tonal Indo-Aryan language, the aspiration has changed into 'breathy voice' in the following vowel. This is confirmed by Author 1 finding about the phonemic contrast of breathy versus modal voice in Gujarati. Thus for the two languages from the same family, the loss of aspiration has resulted in the development of either tone or breathy voice respectively.

Given the complex state of language contact in the region there is also another possibility, namely, both pitch and phonation are involved in the phonemic contrast. For example, Burushaski, an isolate language, is totally different from other languages of the region, yet its tone system is like Shina-type languages of the region due to its geographical contact with Shina (Baart, 2003). Urdu is the national and official language of Pakistan and is spoken by Punjabi and Pahari speakers as a lingua franca. According to Hussain (2010) and M. Ohala, (1991), Urdu still retains voiced aspirates. Punjabi speakers produce Urdu voiced aspirated cognates with low tones. For instance, [ $b^{h} \mathrm{ul}$ ] 'forget' is pronounced as [pòl] 'forget'. The phonemic inventory of Pahari also has no voiced aspirates, and like Punjabi, uses the Low tone for the Urdu voiced cognates (Author 1). For example, [ $\mathrm{b}^{\mathrm{h}} \mathrm{uk}$ ] 'hunger' is pronounced as [pòk] 'hunger'. The close contact (via bilingualism) with a language that is only remotely related means that certain phonetic features of that language are possibly borrowed into the native language. In particular, the voice aspiration in cognates could be passed on to Pahari as breathy voice on vowels (together with tone).

The present study is a comprehensive acoustic analysis of the suprasegmental contrast in Pahari with the goal of testing the above-mentioned possibilities. In summary, the possibilities 
are that the contrast is purely tonal, purely phonational, or both tonal and phonational and involve duration and intensity as well. Thus the following specific research questions are addressed:

1. Does the three-way suprasegmental contrast in Pahari involve $\mathrm{F}_{0}$, phonation type, or both?

2. Are there other phonetic properties, such as duration and intensity, that also correspond to the three-way contrast?

3. Are these contrastive properties, if found, evenly distributed across the contrastive categories?

\section{METHODS}

\section{Participants}

Eight native speakers of Pahari, born and raised in the Pahari-speaking area in the Pakistan-administered part of Kashmir, participated in this study. All the participants were educated in different subjects from Azad Jammu and Kashmir and Pakistan and moved to Nottingham, United Kingdom for higher studies during 2007-2010. Their average age was 35, ranging from 32 to 39 , and none had reported any speech or hearing impairment.

\section{Stimuli}

A list of minimal triplets containing the targeted contrast was prepared. A set of monosyllabic triplet words, forming a corpus of 120 tokens ( 3 words $\times 5$ repetitions $\mathrm{x} 8$ speakers), were written on cards in Urdu characters and the order of their appearance were randomized. The participants read them aloud naturally at a normal speed. Each word was repeated five times, and presented in random order blocked by repetitions.

TABLE I. Minimally contrastive triplet words for recording

\begin{tabular}{|c|c|c|}
\hline Low Tone & Mid Tone & High Tone \\
\hline pa (rate/price) & pa (quarter) & pa (filth of cow/buffalo) \\
\hline
\end{tabular}

\section{Recording}

Recording took place in a quiet room at the home of one of the participants in Nottingham, United Kingdom. Prior to recording, the procedure was explained to all the subjects in a brief practice session. Recordings were completed in two days; each session lasted around 30 minutes. The material was recorded at a sampling rate of $44100 \mathrm{~Hz}$ using Praat (Boersma and Weenik 2010) running on a laptop computer. Each subject was seated in front of the laptop screen 
wearing a head-mounted condenser microphone (Countryman Isomax hypercardiod) which was approximately two inches away from the subject's mouth. The target monosyllabic words were presented on a flash card and the subject was instructed to read each word as naturally as possible. During the recording, the speakers flipped the flash cards themselves and read the words at their own pace.

\section{Measurements}

To determine the acoustic cues associated with the three-way suprasegmental contrast in Pahari, measurements that are detailed and indicative of the underlying properties of the phonetic categories need to be used. To this end a set of measurements of pitch, duration, intensity and voice quality based on findings of previous studies was carefully selected. For pitch, the most informative measurement is continuous $\mathrm{F}_{0}$ contours (Author 2, 1997). In addition, measurements such as mean $\mathrm{F}_{0}$, final $\mathrm{F}_{0}$ and final $\mathrm{F}_{0}$ velocity (hereafter final velocity) have also been used. The last two measurements were developed in a number of recent studies (Liu et al., 2013)and were based on findings that tones are produced as unidirectional movements toward their underlying targets in the tone-bearing syllable (Author 2,2001 ). As a result, the $\mathrm{F}_{0}$ contours and its velocity trajectory near the end of the syllable would most closely approximate the underlying pitch target of the tone.

For voice quality, the most widely used acoustic measure is $\mathrm{H} 1-\mathrm{H} 2$, which is the amplitude difference between the first and second harmonics of the vowel. H1-H2 has been shown to correlate with Open Quotient (OQ) (Holmberg et al., 1995) and has been found to significantly distinguish contrastive phonations in many studies (Andruski and Ratliff, 2000; DiCanio, 2009; Esposito, 2010; Garellek and Keating, 2011; Gordon and Ladefoged, 2001; Holmberg et al., 1995; Kreiman et al., 2007; Miller, 2007; Wayland and Jongman, 2003). In addition, H1-A1, H1A2, H1-A3, which measure the amplitude of the first harmonic $(\mathrm{H} 1)$ relative to the amplitudes of first (A1), second (A2), and third (A3) formants respectively, are also frequently used (Blankenship, 2002; DiCanio, 2009; Esposito, 2006, 2010; Garallek and Keating, 2010; Kuang, 2011; Wayland and Jonhman, 2003). These measurements reflect the relative energy of higher frequencies in the spectrum which is in turn related to the closing velocity of the vocal folds, the presence of the posterior glottal opening, and the simultaneity of ligamental closure (Hanson et al., 2001).

Another widely used measurement is Cepstral Peak Prominence (CPP) defined as peak harmonic amplitude normalized for overall amplitude (Hillenbrand et al., 1994). CPP is predicted to be lower in breathy phonation due to added noise by increased air flow and has been found to be effective in distinguishing breathy from non-breathy voice qualities (Andruski, 2006; Andruski and Ratliff, 2000; Blankenship, 2002; Esposito, 2006, 2010; Wayland and Jongman, 2003).

To inspect these acoustic properties ProsodyPro (Author 2, 2013), a script running under Praat, was used to generate the following measurements:

- Continuous $\mathrm{F}_{0}(\mathrm{~Hz})-\mathrm{F}_{0}$ as a function of time

- Final $\mathrm{F}_{0}(\mathrm{~Hz} / \mathrm{st})-\mathrm{F}_{0}$ taken at $30 \mathrm{~ms}$ before syllable offset

- Final velocity (st/s) - Instantaneous $\mathrm{F}_{0}$ velocity taken at $30 \mathrm{~ms}$ before syllable offset 
- $\quad \mathrm{H} 1-\mathrm{H} 2(\mathrm{~dB})$ - Difference in amplitude between the first and second harmonics

- $\mathrm{H}_{1}$ *-H2$_{-}$* $(\mathrm{dB})$ - Difference in amplitude between the first and second harmonics, corrected using the formula of Iseli, Shue and Alwan (2007). This measurement is included to test its effectiveness compared to $\mathrm{H} 1-\mathrm{H} 2$.

- H1-A1 (dB) - Difference in amplitude between the first harmonic and first formant

- H1-A3 (dB) - Difference in amplitude between the first harmonic and third formant

- CPP - Cepstral Peak Prominence (Hillenbrand et al., 1994)

- Duration (ms) - Duration of tone-bearing syllable

- Mean intensity $(\mathrm{dB})$ - Intensity averaged across the duration of the vowel

ProsodyPro requires users to manually mark the time intervals where measurements are to be taken, and it allows users to manually rectify markings of vocal periods, based on which continuous $\mathrm{F}_{0}$ as well as single-value $\mathrm{F}_{0}$ measurements are taken (Author 2, 2013). This guarantees that the obtained $\mathrm{F}_{0}$ contours are as detailed as possible.

\section{RESULTS}

\section{Fundamental frequency}

Figure 1 displays mean $\mathrm{F}_{0}$ contours of the syllable /pa/ in three tones. Each curve was obtained by averaging the $\mathrm{F}_{0}$ and time values of ten evenly spaced points across the duration of the syllable. As can be seen, the $\mathrm{F}_{0}$ contours are distinct across the three categories in terms of height, shape and duration. Overall, the three tones show relatively high, mid and low $\mathrm{F}_{0}$ except that the Low tone bounces back in its course and ends higher than both the High and Mid tones. The mean $\mathrm{F}_{0}$ values are $160 \mathrm{~Hz}, 149 \mathrm{~Hz}$ and $139 \mathrm{~Hz}$ for High, Mid and Low tones respectively. A one-way repeated measures ANOVA with mean $\mathrm{F}_{0}$ as dependent variable and tone as the independent variable showed a significant effect of tone $(\mathrm{F}(2,7)=19.875, p<0.0001)$. A Bonferroni post-hoc test showed that all three tones are significantly different from each other in terms of mean $\mathrm{F}_{0}$.

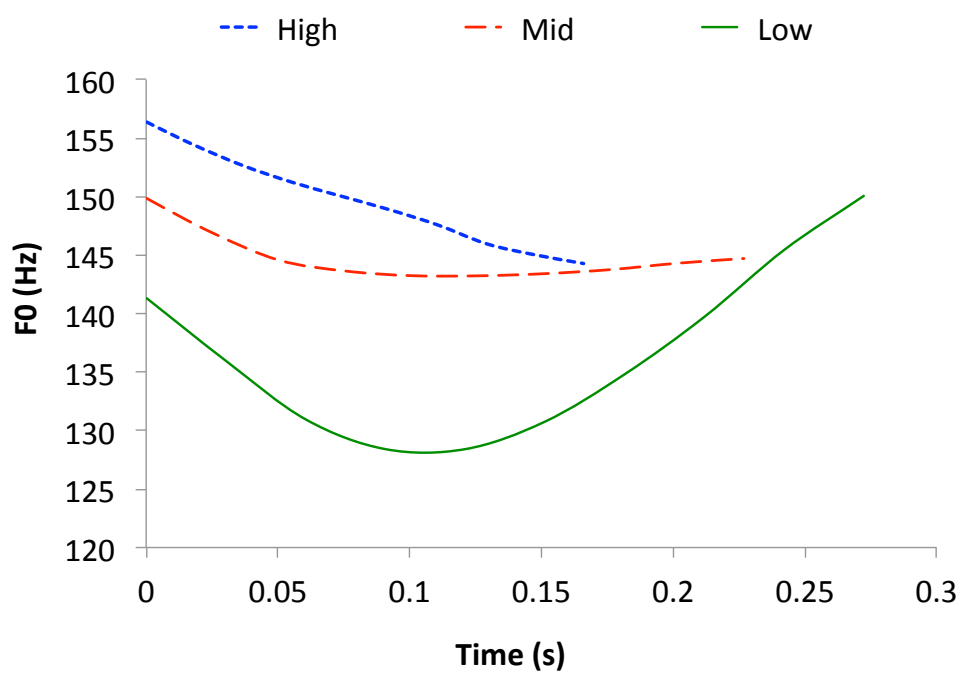


FIG.1. Mean $\mathbf{F}_{\mathbf{0}}$ contours of the three Pahari tones, each consisting of 10 evenly spaced points averaged across five repetitions produced by each of the eight speakers. The X-axis shows time values corresponding to each of $\mathrm{F}_{0}$ points, also averaged across the tokens.

Figure 1 also shows that the three tones High, Mid and Low have falling, level and rising trends, respectively. The High tone starts with a high $\mathrm{F}_{0}(157 \mathrm{~Hz})$ but gradually drops across the syllable $(146 \mathrm{~Hz})$. The Mid tone starts at a slightly lower $\mathrm{F}_{0}(150 \mathrm{~Hz})$, falls to the lowest point of $144 \mathrm{~Hz}$ and then remains stable until the end at $147 \mathrm{~Hz}$. The Low tone starts with the lowest $\mathrm{F}_{0}$ $(142 \mathrm{~Hz})$ of the three tones, falls to the lowest point at $128 \mathrm{~Hz}$ and then rises sharply to $154 \mathrm{~Hz}$ at the end of the syllable.

To further establish the full configuration of the pitch patterns, the average final $\mathrm{F}_{0}$ is plotted against final velocity for all speakers (Figure 2). As can be seen, the Low tone tokens are clustered at the right end of the graph with positive final velocity. This indicates that they all have rising $\mathrm{F}_{0}$ toward the end of the syllable. In contrast, the final velocities of the High tone tokens are distributed toward the left end of the $\mathrm{x}$-axis with none of them showing positive velocity values. This indicates that the High tone is mostly falling toward the end of the syllable. The Mid tone tokens are distributed in the middle of the y-axis, left to the zero line, indicating a near-level pitch toward the end of the syllable.

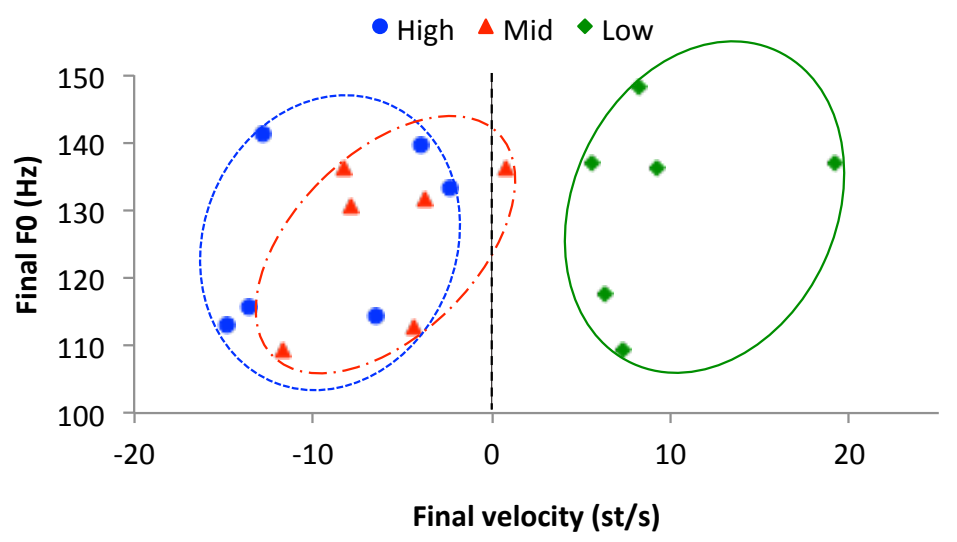

FIG. 2. 2Distribution of final $\mathbf{F}_{\mathbf{0}}$ (y-axis) over final velocity (x-axis) by eight speakers. The ellipses were manually drawn to encircle all the speaker-specific means of the same tone.

A one-way repeated measures ANOVA indicated a significant effect of tone on final velocity $[\mathrm{F}(2,7)=35.52, p<0.0001]$. A Bonferroni post-hoc test showed that final velocity of the Low tone is significantly different from those of the High $(p<0.0001)$ and $\operatorname{Mid}(p<0.0001)$ tones, while no significant difference was found in final velocity between the High and Mid tones.

\section{Duration}

The mean durations of the three tones are plotted in Figure 3. A one-way repeated measures ANOVA and a Bonferroni post-hoc test were conducted to check the effect of tone on 
the duration of the tone-carrying vowels. There is a significant effect of tone on duration ( $p=$ 0.0015). The post-hoc test shows that the High tone is significantly shorter than the Mid and Low tones ( $\mathrm{p}=0.0012$ and $p=0.0014$, respectively), but there was no significant difference between the Mid and Low tones.

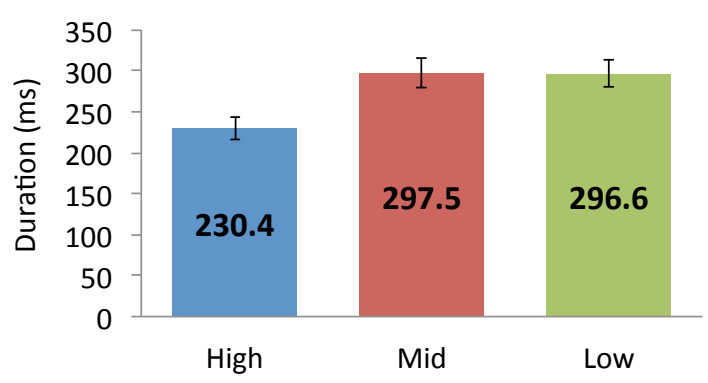

FIG. 3. Mean duration (ms) of the High, Mid and Low tones with standard errors.

\section{Intensity}

Figure 4 displays mean intensity of the three tones. A one-way repeated measures ANOVA shows a significant effect of tone on the intensity of the tone-carrying vowels $[\mathrm{F}(2,7)=11.862$, $p=0.0010]$. A Bonferroni post-hoc test indicated that the intensity of the Low tone was significantly lower than both of the remaining tones. The other tones were not found to be different in intensity.

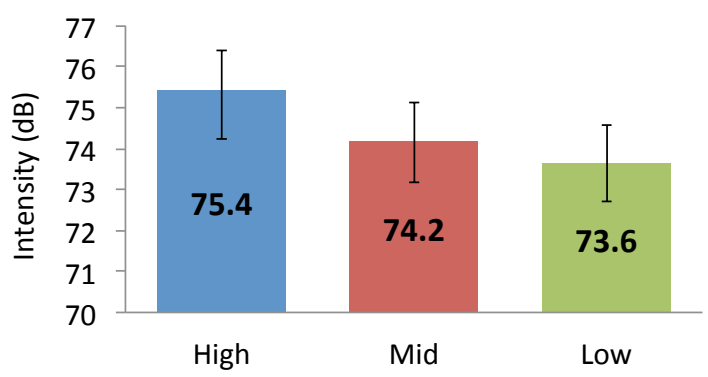

FIG. 4. Mean intensity of the High, Mid and Low tones with standard errors.

\section{Phonation}

Table II is a summary of the results of one-way repeated measures ANOVAs comparing the three tones in various voice quality measurements. Columns 2-4 list the direction of the differences for each measurement; the $\mathrm{F}$ values and $p$ values with tone as the independent variable.

TABLE II. Results of one-way repeated measures ANOVAs on all acoustic measurements for voice quality.

\begin{tabular}{|l|l|l|l|}
\hline Measurements & Direction of difference & $\mathrm{F}(2,7)$ & $p$ \\
\hline
\end{tabular}




\begin{tabular}{|l|l|c|c|}
\hline H1-H2 & Higher for Low tone & 4.567 & $\mathbf{0 . 0 2 9 7}$ \\
\hline H1 ${ }^{-H} 2 *$ & Lower for Low tone & 0.534 & 0.5979 \\
\hline H1-A1 & Lower for Low tone & 2.57 & 0.112 \\
\hline H1-A3 & Lower for Low tone & 11.21 & $\mathbf{0 . 0 0 1 2}$ \\
\hline CPP & Lower for Low tone & 22.90 & $\mathbf{0 . 0 0 0 1}$ \\
\hline
\end{tabular}

Figure 5 shows bar graphs of $\mathrm{H} 1-\mathrm{H} 2$ (left) and $\mathrm{H} 1 *-\mathrm{H} 2 *$ (right). As can be seen, $\mathrm{H} 1-\mathrm{H} 2$ has a higher value for the Low tone than for the Mid and High tones. A post-hoc Bonferroni test showed that the Low tone was significantly different from both the High and Mid tones in terms of H1-H2. However, there was no significant difference between the Low and High tones or between the High and Mid tones. This indicates that the Low tone has a steeper downward spectral slope than the other two tones at the low-frequency end of the spectrum. For H1*-H2*, as shown in the graph on the right in Figure 5, there was no significant effect of tone.
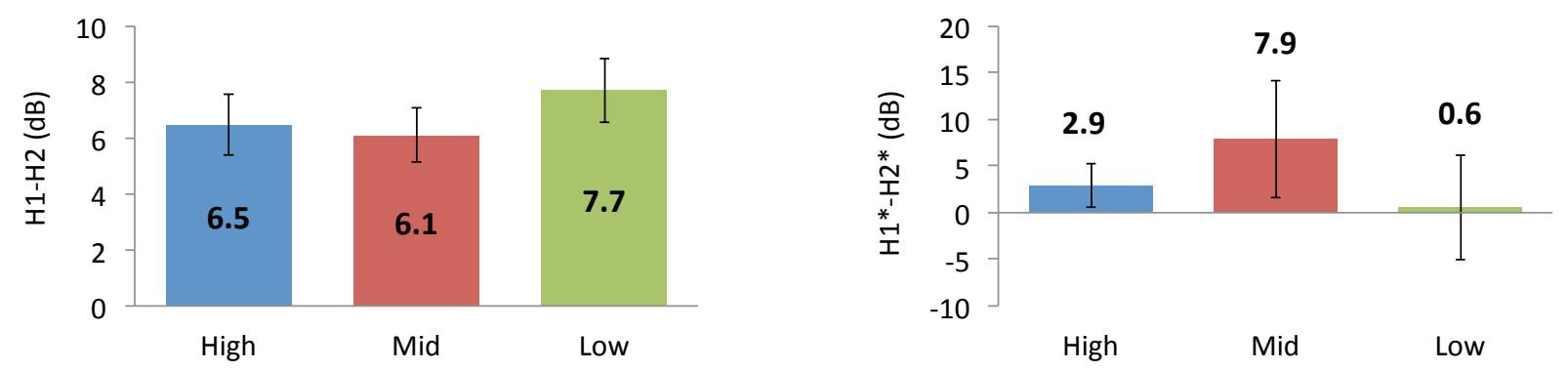

FIG. 5. Bar graphs of $\mathrm{H} 1-\mathrm{H} 2$ (left) and $\mathrm{H} 1 *-\mathrm{H} 2 *$ (right) for High, Mid and Low tones with standard errors.

It was surprising that there was no significant tone effect on $\mathrm{H} 1 *-\mathrm{H} 2 *$. One possibility is that there was an interaction between voice quality and vowel production. That is, the incomplete closure of the glottis in breathy phonation may have made it difficult to maintain transglottal pressure needed for vocal vibration. One way to sustain sufficient transglottal pressure is to lower the larynx to reduce the subglottal cavity (Hanson and Stevens, 2002). However, lowering the glottis also lengthens the vocal tract, which would lower all the formants, other things being equal (Stevens, 1998). To see if this was the case FormantPro, a Praat script developed by the second author for systematic formant analysis (Author 2, 2018), was used to track continuous formants during the three tones. Figure 8 displays time-normalized formant trajectories averaged across all the tokens produced by all the speakers. As can be seen, toward the end of the vowel the first three formants (i.e. those used in the $\mathrm{H} 1 *-\mathrm{H} 2 *$ correction) were all lower in the Low tone than in the other two tones. A set of one-way repeated measures ANOVAs were performed on the last three of the 20 time-normalized formant measurements and the results are shown in TABLE III. There was a gradual increase in the significance level of all three formants, and for F2 all three points showed significant difference. Such formant variations therefore may have interfered with the correction in the calculation of $\mathrm{H} 1 *-\mathrm{H} 2 *$. Note that the need for the formant correction is to compensate for the spectral variations across vowels of varying height or frontness (Iseli et al., 2007). In the present study, because the tone-carrying vowels were kept constant across the hypothetical tones, the need to make the correction was largely circumvented. 
Keeping the vowel constant when comparing spectral properties associated with voice quality may therefore be a better way of controlling for spectral variability across vowels in controlled experimental studies.

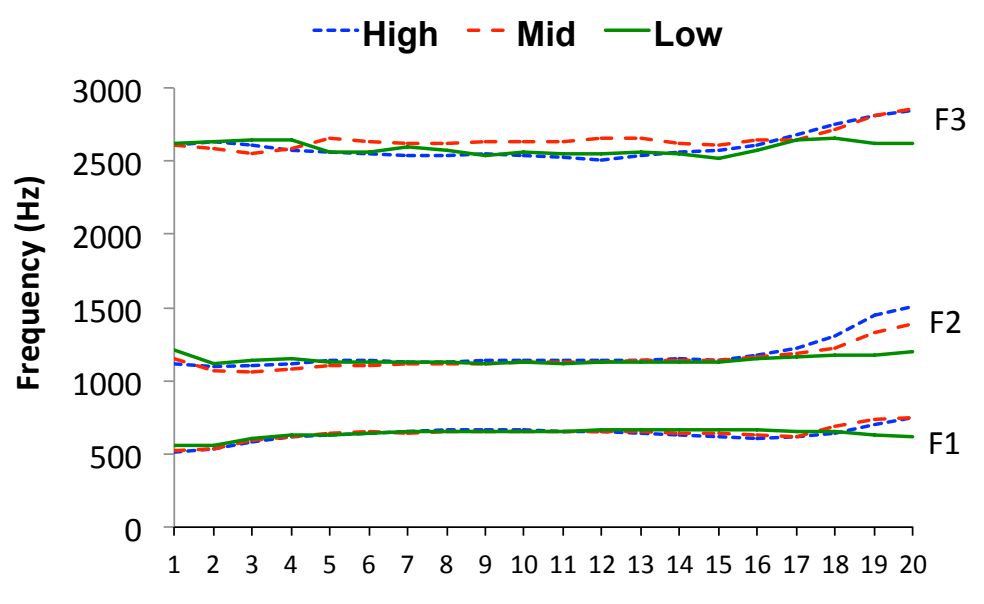

Normalized time

FIG. 6. Time-normalized trajectories of the first three formants of the vowels carrying the High, Mid and Low tones

TABLE III. Results of one-way repeated measures ANOVAs on the last three points of F1, $\mathrm{F} 2$ and $\mathrm{F} 3$ trajectories across three tones.

\begin{tabular}{|l|l|l|}
\hline Formant points & $\mathrm{F}(2,7)$ & $P$ \\
\hline F1-18 & 0.73 & 0.4992 \\
\hline F1-19 & 1.54 & 0.249 \\
\hline F1-20 & 2.54 & 0.1146 \\
\hline F2-18 & 3.79 & $\mathbf{0 . 0 4 8 3}$ \\
\hline F2-19 & 5.23 & $\mathbf{0 . 0 2 0 1}$ \\
\hline F2-20 & 6.97 & $\mathbf{0 . 0 0 7 9}$ \\
\hline F3-18 & 1.23 & 0.3226 \\
\hline F3-19 & 3.72 & 0.0505 \\
\hline F3-20 & 4.64 & $\mathbf{0 . 0 2 8 5}$ \\
\hline
\end{tabular}

Figure 7 displays bar graphs of H1-A1 and H1-A3. There is no significant effect of tone on $\mathrm{H} 1-\mathrm{A} 1[\mathrm{~F}(2,7)=2.57, p=0.112]$. However, the effect of tone is significant on $\mathrm{H} 1-\mathrm{A} 3[\mathrm{~F}(2,7)=$ $11.21, p=0.0012]$. The direction of the difference between the means, as shown in Figure 7 right, was unexpected, because the Low tone actually shows lower H1-A3 than the other two tones. This suggests a shallower rather than steeper spectral slope in the higher frequency regions. 

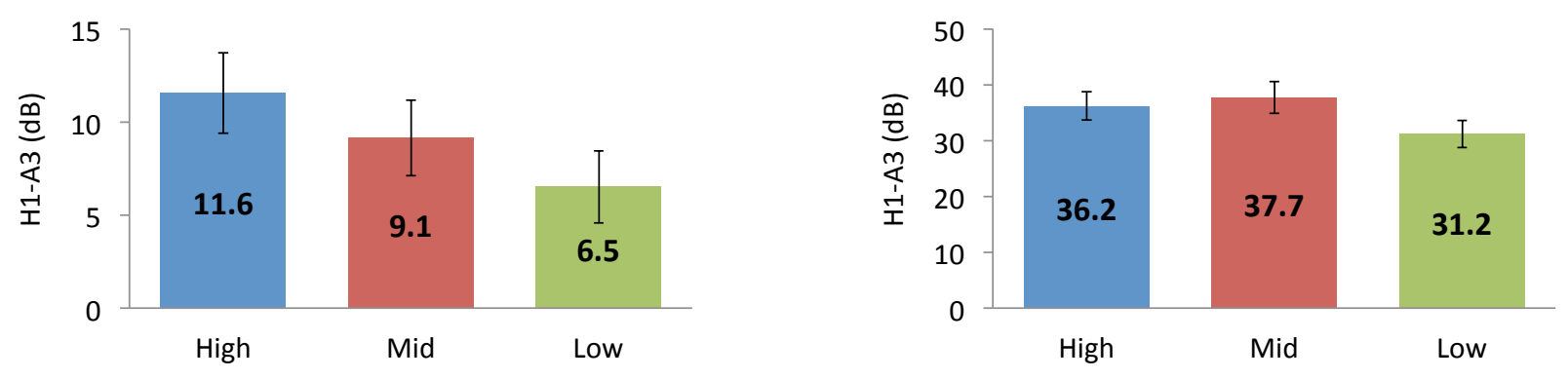

FIG. 7. Bar graphs of H1-A1 (left) and H1-A3 (right) for High, Mid and Low tones with standard errors.

Finally, Figure 8 displays mean values of CPP across the three tones. Consistent with H1$\mathrm{H} 2$, CPP shows lower values for the Low tone than for the other two tones. A one-way repeated measures ANOVA shows a significant effect of tone on $\mathrm{CPP}[\mathrm{F}(2,7)=22.904, p<0.0001]$. A post-hoc Bonferroni test found significant differences in CPP between the Low tone and both the Mid and High tones, but no difference between the Mid and High tones.

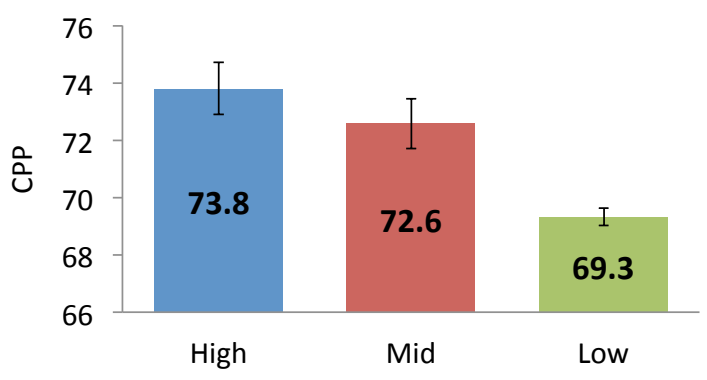

FIG. 8. Bar graphs of CPP for the High, Mid and Low tones with standard errors.

Overall, the analysis of voice quality indicates that there is phonation variation associated with the tones. Specifically, the Low tone seems to have a breathy voice as indicated by H1-H2 and CPP, while the High and Mid tones have no difference in voice quality. The higher values of $\mathrm{H} 1-\mathrm{H} 2$ found in the Low tone indicates a greater tilt in the low end of the spectrum and the lower value of CPP in the Low tone indicates a smaller harmonic-to-noise ratio than in the other two tones.

\section{GENERAL DISCUSSION}

The results of the detailed acoustic analyses have provided answers to the three research questions raised at the outset of this study. The $\mathrm{F}_{0}$ analysis has confirmed the tonal nature of the three suprasegmental categories, namely the High, Mid and Low tones. The average $\mathrm{F}_{0}$ difference of the three tones is not as large as in languages like Mandarin (Author 2,1997), but relatively small differences in fundamental frequency is not uncommon in contrastive tones in other languages (Barry and Blamey, 2004). The three tones are significantly different from each other in terms of their final velocity, showing falling, level and rising patterns for the High, Mid and Low tones respectively. Assuming that final velocity is indicative of direction of underlying pitch target (Author 2, 2014), it can be concluded that the three lexical tones in Pahari can be characterized as High-falling, Mid-level and Low-rising. The analysis has further shown that the High tone is shorter than the Mid and Low tones, although there are no significant three-way 
durational differences. The $\mathrm{F}_{0}$ and duration patterns are consistent with previous studies conducted by Author 1 (2017).

Intensity is also found to be lower in the Low tone than the other two tones. This could be due to both the well-known correlation of $F_{0}$ and intensity (Stevens, 1998) and the breathiness associated with the Low tone to be discussed next.

The multiple measurements taken in this study to assess voice quality have provided some evidence that the Low tone is produced with breathy phonation. This is demonstrated by significantly higher H1-H2 and lower CPP in the Low tone compared to the other two tones, measurements that have been shown to be the most reliable indicators of breathy phonation (Blankenship, 2002; Espisito, 2006). Interestingly, while phonation can only help to distinguish the Low tone from the other two tones, duration helps to distinguish the High tone from the Mid and Low tones. Thus there appears to be a near complementary distribution of the non- $\mathrm{F}_{0}$ cues for the three tones of Pahari (see Table IV).

TABLE IV. Summary of the results of all the acoustic measures.

\begin{tabular}{|c|c|c|c|}
\hline $\begin{array}{ll} & \text { Tone } \\
\text { Measurement } & \\
\end{array}$ & High & Mid & Low \\
\hline $\mathrm{F}_{0}$ & high & mid & low \\
\hline Final velocity & falling & level & rising \\
\hline Duration & shorter & longer & longer \\
\hline Intensity & higher & higher & lower \\
\hline Phonation & modal & modal & breathy \\
\hline
\end{tabular}

As can be seen in Table IV, the High tone is associated with high $\mathrm{F}_{0}$, falling final velocity, shorter duration, higher intensity and modal phonation. The Mid tone is associated with mid $\mathrm{F}_{0}$, level final velocity, longer duration, higher intensity and modal phonation. The Low tone is associated with low $\mathrm{F}_{0}$, rising final velocity, longer duration, lower intensity and breathy phonation. Thus the five acoustic cues jointly contribute to the lexical tonal contrast in Pahari. The overall height of $F_{0}$ is the most salient, as it is significantly different across all three categories. Final velocity helps to distinguish the Low tone from the other two tones. Phonation and intensity help to distinguish the Low tone from the Mid tone and duration helps to distinguish the High tone from the Mid and Low tones.

The suprasegmental lexical contrasts in Pahari as shown in Table IV may raise interesting questions, however, about the notion of distinctive features (Jakobson, Fant and Halle, 1963). That is, the concept of distinctive features assumes that the smallest units of lexical contrast in speech are not phonemes, but properties that serve to make them distinctive. More importantly, it is assumed that breaking phonemes down into distinctive features further reduces the number of basic contrastive units, which is thus consistent with the principle of minimal redundancy in phonetic representation. Based on this principle, distinctive tones in a language should involve only contrastive pitch variations, and distinctive phonation types should involve only contrastive voice quality. The present findings about Pahari tones, however, seem to be inconsistent with the minimal redundancy principle. Instead, all the acoustic cues found to be significant in the present 
data seem to work together to maximize the contrastiveness of a suprasegmental category, although the contribution of $\mathrm{F}_{0}$ is dominant. This multiplicity of cues makes sense in terms of speech communication, because what is really critical is for the speaker to indicate to the listener in the clearest manner possible which particular word is being spoken. Thus the contrast is ultimately a lexical one as opposed to a featural one for the sake of economy of representation.

It has been proposed, however, that certain phonetic properties act as enhancing gestures that serve to enhance the saliency of the defining feature (Stevens and Keyser, 2010). Following this suggestion, the enhancing gesture should at least be related to the core properties of the defining feature. In the case of the Low tone, a creaky phonation is likely used as an enhancing gesture, as it is a natural consequence of the low $\mathrm{F}_{0}$ articulation (Klatt \& Klatt, 1990). However, the Pahari Low tone is associated with breathy phonation, and the source of the breathiness may be the voiced aspiration of either the parent language or a second language. It is therefore unclear that the breathy phonation is there to enhance the low $\mathrm{F}_{0}$. Instead, the hypothesis that both of features, together with duration and intensity, contribute to marking the lexical contrast in addition to the contrasts marked by consonants and vowels is more plausible. More importantly, in neither of the two accounts does the phonetic realization of a phonemic (tonal in this case) contrast seem to follow the principle of economy of featural representation.

Theoretical issues notwithstanding, the present study is pioneering in the research of suprasegmental lexical contrasts in the languages of the area. There have not been any acoustic studies conducted previously either on other related languages in the region nor on the role of acoustic measures other than $\mathrm{F}_{0}$ and duration. The finding that Pahari is likely a full-fledged tonal language points to the need to look into the tonal aspects of other related languages (Baart, 2003). And the methology used in the present study is potentially applicable to future studies.

The current study also has some limitations that need to be addressed in the future. A major limitation is that only monosyllabic words in isolation (i.e., without carrier) have been analysed. Future studies need to investigate disyllabic or multisyllabic words and the role of context in order to examine a broader range of tonal and phonational phenomena. In addition, crosslinguistic comparisons on tonal languages of the region using all the parameters employed in the present study may help to establish more generalised phonetic trends in the region.

\section{Conclusion}

It can be concluded from the results of the present study that Pahari is tonal at the lexical level, with three distinct tones, High, Mid and Low, characterized by high-falling, mid-level and low-rising $\mathrm{F}_{0}$ contours respectively. These $\mathrm{F}_{0}$ patterns are accompanied by near-complementary distribution of secondary cues, the Low tone being separated from the other two tones with breathy phonation, and the High tone separated from the other two tones with shorter duration. Thus the five acoustic cues jointly contribute to the lexical tonal contrast in Pahari. The height of $\mathrm{F}_{0}$ is the most salient. Final velocity also helps to distinguish the Low tone from the other two tones. Intensity and phonation help to distinguish the Low tone from the Mid tone. And duration helps to distinguish the High tone from the Mid and Low tones.

This study is the first comprehensive investigation of tonal aspects of a language in the area, where many languages are either closely related or spoken as a second language in daily life. The finding that Pahari shows a Punjabi-type three-way tonal contrast means that the 
methodology of multidimensional analysis of tone used in the study can be applied to other tonal languages in the area. The current findings also raise interesting questions about the notion of distinctive features as well as the principle of economy of representation behind it, suggesting a need for further theoretical exploration.

\section{References}

Andruski, J. E., Ratliff, M., 2000. Phonation types in production of phonological tone: the case of Green Mong. Journal of International Phonetic Association. 30, 37-61.

Baart, J. L.G., 2003. Tonal Features in languages of northern Pakistan. In: Pakistani languages and society: problems and prospects, edited by Joan. L.G. Baart and Ghulam Hyder Sindhi. National Institute of Pakistan Studies and Summer Institute of Linguistics, Islamabad, pp. 132-144.

Barry, J. G., Blamey, P. J., 2004. The acoustic analysis of tone differentiation as a means for assessing tone production in speakers of Cantonese. The Journal of the Acoustical Society of America.116, 1739-1748.

Belotel-Grenie, A., Grenie, M., 1994. Phonation type analysis in Standard Chinese. In: Proceedings of International Conference on Spoken Languages Processing, Yokohama, Japan, pp. 343-346.

Bhatia, T. K., 1975. The evolution of tones in Punjabi. Studies in the Linguistic Sciences. 5(2), 12-24.

Blankenship, B., 2002. The timing of nonmodal phonation in vowels. Journal Phonetics 30, 163-191.

Boersma, P., 2001. Praat, a system for doing phonetics by computer. Glot International. 5(9), 341-345.

DiCanio, C. T., 2009. The phonetics of register in Takhian Thong Chong. Journal of International Phonetic Association. 39, 162-188.

Esposito, C.M., 2006. The effects of linguistic experience on the perception of phonation. Ph.D Dissertation, UCLA.

Esposito, C. M., 2010. Variation in contrastive phonation in Santa Ana Del Valle Zapotec. Journal of International Phonetic Association. 40, 181-198.

Faytak, M., Yu, A.C.L., 2011. A typological study of the interaction between level tones and duration. In: Proceedings of ICPhS XVII.

Garellek, M., Esposito, C. M., Keating, P., Kreiman, J., 2012. Perception of spectral slopes and tone identification in White Hmong. UCLA Working Papers in Phonetics. 110, 24-45.

Garellek, M., Keating, P., 2011. The acoustic consequences of phonation and tone interactions in Jalapa Mazatec. Journal of the International Phonetic Association. 41, 185-205.

Gill, H. S., Gleason, H. A., 1972. The salient features of the Punjabi language. Pakha Sanjam. 5, $1-50$

Gordon, M., Ladefoged, P., 2001. Phonation types: a cross-linguistic overview. Journal of Phonetics. 29, 383-406.

Hanson, H. M., Stevens, K. N., Kuo, H. K., Chen, M. Y., Slifka, J., 2001. Towards models of phonation. Journal of Phonetics. 29, 451-480.

Hillenbrand, J., Cleveland, R. A., Erickson, R. L., 1994. Acoustic correlates of breathy vocal Quality. Journal of Speech, Language, and Hearing Research. 37, 769-778. 
Holmberg, E. B., Hillman, R. E., Perkell J. S., Guiod, P. C., Goldman, S. L., 1995. Comparisons among aerodynamic, electroglottographic, and acoustic spectral measures of female voice. Journal of Speech, Language, and Hearing Research. 38, 1212-23.

Hombert, J. M., Ohala, J., Ewan, W., 1979. Phonetic explanation for the development of tones. Language. 55, 37-58.

Hussain, S., 2010. Phonetic correlates of Urdu lexical stress. National Urdu Academy, Islamabad.

Iseli, M., Shue, Y.L., Alwan, A., 2007. Age, sex, and vowel dependencies of acoustic measures related to the voice source. Journal of Acoustical Society of America. 121, 2283-2295.

Jakobson, R., Fant, G., Halle, M., 1963. Preliminaries to Speech Analysis. MA: MIT Press (Originally published in 1951), Cambridge, p. 64.

Klatt, D. H., Klatt, L. C., 1990. Analysis, synthesis, and perception of voice quality variations among female and male talkers. Journal of Acoustical Society of America. 87, 820-857.

Kreiman, J., Gerratt, B., Antonanzas-Barroso, N., 2007. Measures of the glottal source spectrum. Journal of Speech, Language, and Hearing Research. 50, 595-610.

Kuang, J., 2011. Production and perception of the phonation contrast in Yi. M.A. thesis, University of California Los Angeles.

Kuo, Y.C., Rosen, S., Faulkner, A., 2008. Acoustic cues to tonal contrasts in Mandarin: implications for cochlear implants. Journal of Acoustical Society of America, 123(5) $2815-2824$.

Ladefoged, P., 1971. Elements of Acoustic Phonetics. University of Chicago Press, Chicago.

Lothers, M., Lothers, L., 2010. Pahari and Pothwari: A sociolinguistics survey. Summer Institute of Linguistics, Islamabad.

Ohala, M., 1991. Phonological areal features of some Indo-Aryan languages. Language Sciences. 13(2), 107-124.

Pike, K. L., 1948. Tone Languages. University of Michigan Press, Michigan.

Silverman, D., 1997. Phasing and Recoverability. Routledge, London.

Stevens, K. N., 1998. Acoustic Phonetics. The MIT Press, Cambridge, MA.

Stevens, K. N., Keyser, S. J., 2010. Quantal theory, enhancement and overlap. Journal of Phonetics. 38(1), 10-19.

Wayland, R., Jongman, A., 2003. Acoustic correlates of breathy and clear vowels: The case of Khmer. Journal of Phonetics. 31, 181-201.

Yip, M. J. W., 2002. Tone. Cambridge University Press, Cambridge.

Zheng, X., 2006. Voice Quality Variation with Tone and Focus in Mandarin. In: The 2nd International Symposium on Tonal Aspects of Languages. La Rochelle, France, pp. 139143. 